\title{
Comparison Microbial Killing Efficacy between Sonodynamic Therapy and Photodynamic Therapy
}

\author{
Nike Dwi Grevika Drantantiyas*a, Suryani Dyah Astuti ${ }^{\mathrm{b}}$, Aulia MT Nasution ${ }^{\mathrm{a}}$ \\ ${ }^{a}$ Magister of Engineering Physics, Institut Teknologi Sepuluh Nopember, Surabaya, Indonesia, \\ bPostgraduate Program in Biomedical Engineering, Airlangga University, Surabaya, Indonesia
}

\begin{abstract}
Biofilm is a way used by bacteria to survive from their environmental conditions by forming colony of bacteria. Specific characteristic in biofilm formation is the availability of matrix layer, known as extracellular polymer substance. Treatment using antibiotics may lead bacteria to be to resistant. Other treatments to reduce microbial, like biofilm, can be performed by using photodynamic therapy. Successful of this kind of therapy is induced by penetration of light and photosensitizer into target cells. The sonodynamic therapy offers greater penetrating capability into tissues. This research aimed to use sonodynamic therapy in reducing biofilm. Moreover, it compares also the killing efficacy of photodynamic therapy, sonodynamic therapy, and the combination of both therapeutic schemes (known as sono-photodynamic) to achieve higher microbial killing efficacy. Samples used are Staphylococcus aureus biofilm. Treatments were divided into 4 groups, i.e. group under ultrasound treatment with variation of 5 power levels, group of light treatment with exposure of 75s, group of combined ultrasound-light with variation of ultrasound power levels, and group of combined lightultrasound with variation of ultrasound power levels. Results obtained for each treatment, expressed in \% efficacy of log $\mathrm{CFU} / \mathrm{mL}$, showed that the treatment of photo-sonodynamic provides greater killing efficacy in comparison to either sonodynamic and sono-photodynamic. The photo-sonodynamic shows also greater efficacy to photodynamic. So combination of light-ultrasound (photo-sonodynamic) can effectively kill microbial biofilm. The combined therapy will provide even better efficacy using exogenous photosensitizer.
\end{abstract}

Keywords: Photodynamic therapy, cahaya, Biofilm, ultrasound, sonodynamic therapy, photo-sonodynamic therapy, sono-photodynamic therapy, efficacy.

\section{INTRODUCTION}

Staphylococcus aureus is bacterial pathogen that one of main caused is chronic infection ${ }^{1,2}$. Their mode of survival to environment's conditions is carried out by forming biofilm ${ }^{3}$. Biofilm is regarded as the phase where bacteria forming their community via the quorum sensing ${ }^{4,5}$. This community will produce layer of matrix called extracellular polymer substance ${ }^{5,6}$ When it formed biofilm, bacteria will be and persistent in compared to previous their planctonic phase ${ }^{7}$. Many antibiotic treatments cannot reduce biofilm, even it might lead to be resistances. Antibiotic resistance is induced by the $\beta$-lactamase enzym and efflux pump. Alternative therapy is able to reduce without make it resistance is photodynamic therapy. ${ }^{8,9}$

Photodynamic therapy has been practicing in reducing microorganism ${ }^{10}$, is kind of therapeutic modality using photosensitizer and light (in certain wavelength) to produce reactive oxygen species (ROS) ${ }^{11}$. Photosensitizer can be endogenously required in the body. One of endogenous photosensitizer is porphyrin. Staphylococci bacteria the most is uroporphyrin $^{12}(89 \%)$ dan coproporphyrin III $(68.3 \%-74.6 \%) .{ }^{13}$ Absorbance spectra of Staphylococcus aureus tend to be around blue wavelength. By activating endogenous photosensitizer in bacteria, interactions between light and photosensitizer material will lead to ROS production ${ }^{14}$. These ROS molecules is toxic in nature and can cause cells apoptosis and necrosis. Deeper penetration of light and photosensitizer into cells regarded as the successful factors to produce greater apoptosis and necrosis. ${ }^{15}$ This deeper penetration is aimed to reduce the target cells in tissue. One of the alternative to achieve this condition is by utilizing ultrasound wave.

Ultrasound has been widely applicated on imaging and medical. Application of ultrasound on therapy is called by Sonodynamic therapy (SDT). ${ }^{16,17}$ It offers advantages in more penetration into biological tissue. The interactions between

\footnotetext{
Second International Seminar on Photonics, Optics, and Its Applications (ISPhOA 2016)

edited by Agus Muhamad Hatta, Aulia Nasution, Proc. of SPIE Vol. 10150, 101501G

(C) 2016 SPIE - CCC code: $0277-786 X / 16 / \$ 18 \cdot$ doi: 10.1117/12.2248503
}

Proc. of SPIE Vol. 10150 101501G-1 